\title{
Primitive neuroectodermal tumor of the kidney at the advanced stage: A case series of eight Chinese patients
}

\author{
QIPENG SUN $^{1 *}$, BIN MIAO $^{2 *}$, XIANGMING LAO $^{3}$, PING YUAN $^{4}$, JIARONG CAI $^{1}$ and HAILUN ZHAN ${ }^{1}$ \\ Departments of ${ }^{1}$ Urology and ${ }^{2}$ Renal Transplantation, Lingnan Hospital, The Third Affiliated Hospital, \\ Sun Yat-sen University, Guangzhou, Guangdong $510530 ;{ }^{3}$ Department of Hepatobiliary Surgery, Cancer Center, \\ Sun Yat-sen University, Guangzhou, Guangdong 510060; ${ }^{4}$ Department of Obstetrics and Gynecology, IVF Center, \\ Sun Yat-sen Memorial Hospital, Sun Yat-sen University, Guangzhou, Guangdong 510120, P.R. China
}

Received March 9, 2016; Accepted February 17, 2017

DOI: $10.3892 / \mathrm{mco} .2018 .1611$

\begin{abstract}
Primitive neuroectodermal tumor (PNET) rarely occurs as a primary renal neoplasm. Renal (r)PNET is a rare but aggressive neoplasm with poor prognosis; the majority of patients are diagnosed as advanced stage at presentation and face a worse prognosis than patients with localized disease. The present study describes the diagnosis and management of eight cases of rPNET at an advanced stage, who were treated at two institutions [Lingnan Hospital (branch of The Third Affiliated Hospital) and the Cancer Center of Sun Yat-sen University, Guangzhou], from December 2004 to January 2013. The clinical and pathological results of all patients were retrospectively obtained. Kaplan-Meier analysis was performed to estimate patient survival. The study cohort comprised five males and three females. Radical nephrectomy was performed in seven cases, while the remaining case only received needle biopsy of the tumor. Five cases received adjuvant chemotherapy, while three received no further treatment after surgery. Of note, one case received cytokine-induced killer (CIK) cell immunotherapy combined with surgery and chemotherapy. The overall median survival was 20 months with a 3 -year survival rate of $25 \%$. The overall survival of the four patients who received adjuvant chemotherapy following surgery was 36 months, compared with 10 months in the three patients without further treatment. The patient who received CIK cell immunotherapy survived for 20 months. Based on the observations of the present and previous studies, surgical excision and chemotherapy
\end{abstract}

Correspondence to: Dr Hailun Zhan, Department of Urology, Lingnan Hospital, The Third Affiliated Hospital, Sun Yat-sen University, 2693 Kaichuang Road, Huangpu, Guangzhou, Guangdong 510530, P.R. China

E-mail: sysusqp@163.com

${ }^{*}$ Contributed equally

Key words: cancer treatment, renal primitive neuroectodermal tumor, immunotherapy, cytokine-induced killer cells are recommended for treating rPNET at advanced stage. Furthermore, the present study was the first to report on CIK cell immunotherapy for a patient with rPNET, indicating that it may be a promising optional treatment. However, further studies are required to validate the benefit of CIK cells and to establish an appropriate immunotherapy protocol.

\section{Introduction}

Primitive neuroectodermal tumor (PNET) is a rare malignancy that is a member of the family of 'small round-cell tumors' and is often classified as central nervous system PNET or peripheral PNET, depending on its site of origin (1). Although PNET has been reported in the brain, ovary and liver (2), renal (r)PNET is rare, with only 120 cases of rPNET reported in the medical literature since its discovery in 1975 (3). rPNET usually affects young adults and features a rapid clinical progression and significant mortality due to late diagnosis, early metastasis and advanced stage at presentation (4). The overall survival of rPNET at the advanced stage is only 15 months, compared with 60 months in patients with localized tumors (5). In spite of common treatments, including surgical excision, chemotherapy and radiotherapy, providing a survival benefit for patients with rPNET at the localized stage, the prognosis at the advanced stage remains poor $(1,6)$. Therefore, novel therapeutic approaches to prolong survival are required, particularly for patients at the advanced stage. Immunotherapy is a novel treatment for various types of tumor, including renal cell carcinoma (RCC), and has achieved encouraging results (7). Numerous studies have demonstrated the anti-tumor properties of cytokine-induced killer (CIK) cells, such as enhanced cytotoxic activity and resistance to Fas-mediated apoptosis (8). To the best of our knowledge, no previous study has reported on CIK cell immunotherapy for patients with rPNET. Between December 2004 and January 2013, eight patients with rPNET at an advanced stage were treated at Lingnan Hospital (branch of The Third Affiliated Hospital) and the Cancer Center of Sun Yat-sen University (Guangzhou, China), of which one case was administered CIK cells, with the aim of improving the long-term survival after having obtained informed consent. The present study describes the 

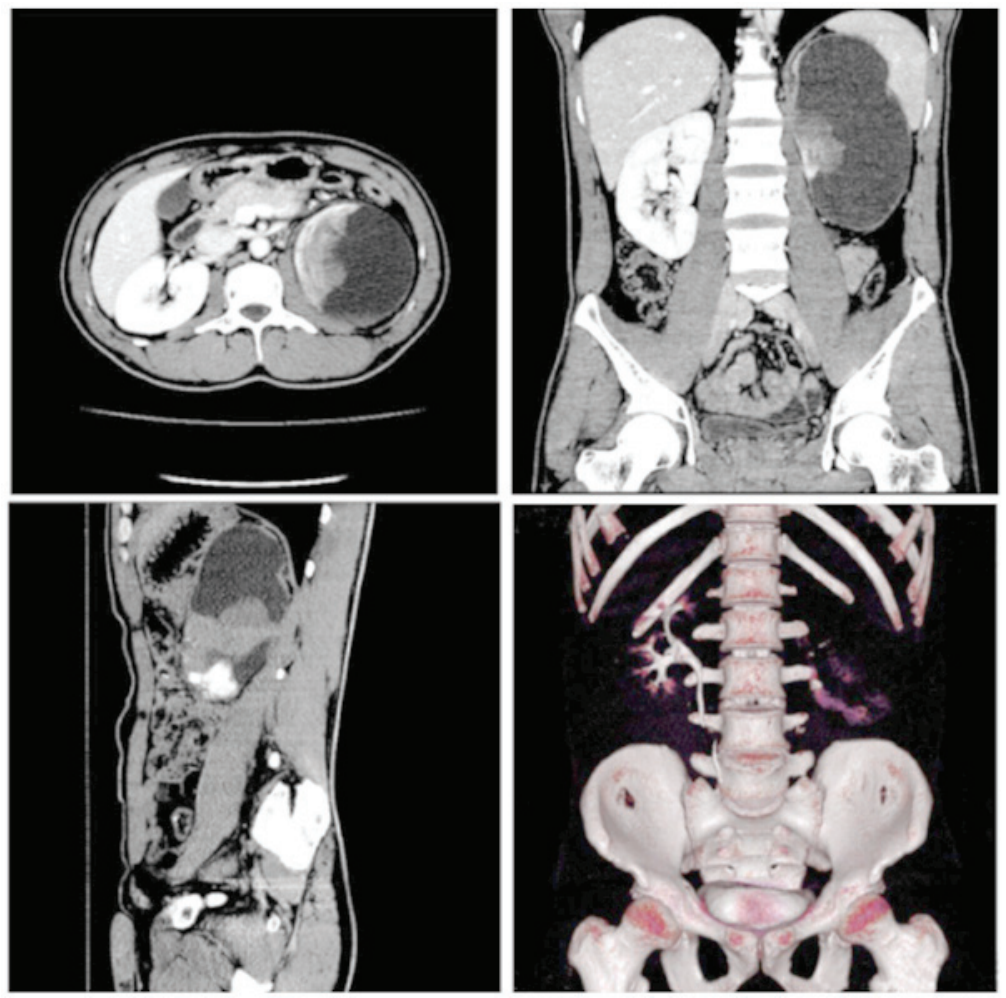

Figure 1. Pre-operative abdominal computed tomography images of case no. 8. A poorly-defined tumor on the lateral side of the left kidney compressed the left pelvis, pancreatic tail and inner side of the spleen. The upper left, upper right and bottom left images show the tumor from different angles, whereas the bottom right image shows a three-dimensional reconstruction of abdomen, revealing that the left tumor invaded the left kidney totally.

pathological and clinical features, as well as the treatment outcomes, of these cases, in addition to a review of the literature on rPNET.

\section{Patients and methods}

Patients. Between December 2004 and January 2013, eight cases of rPNET at the advanced stage were treated at two institutions [Lingnan Hospital (branch of The Third Affiliated Hospital) and the Cancer Center of Sun Yat-sen University, Guangzhou]. The cohort comprised five males and three females with a median age of 34 years (range, $17-45$ years) at presentation. All of the patients complained of a palpable abdominal mass; furthermore five cases had abdominal pain and one case had edema in the lower limbs. Patient evaluation included history, physical examination, complete blood count, renal and liver function tests, chest X-ray, computed tomography $(\mathrm{CT})$ scan of the abdomen and radionuclide renography. On the CT scan with heterogeneous contrast enhancement, the tumors ranged from $4-22 \mathrm{~cm}$ in size (median, $11 \mathrm{~cm}$ ) and few showed calcified areas (Fig. 1). Seven cases received radical surgery, while the remaining case was only subjected to needle biopsy of the tumor due to tumor invasion of the inferior vena cava. Five cases received adjuvant chemotherapy, while three cases received none. Surgical treatment consisted of radical nephrectomy, as well as resection of parts of regional organs and lymph nodes as required.

CIK cell preparation. CIK cells were prepared as described in a previous study (9). In brief, peripheral blood mononuclear cells (PBMCs) were collected from the patient and cultured in medium containing $50 \mathrm{ng} / \mathrm{ml}$ anti-CD3 antibody, $100 \mathrm{U} / \mathrm{ml}$ recombinant human interleukin (IL)- $1 \alpha$ and $1,000 \mathrm{U} / \mathrm{ml}$ interferon (IFN) $\gamma$ at $37^{\circ} \mathrm{C}$ in an atmosphere containing $5 \% \mathrm{CO}_{2}$ for $24 \mathrm{~h}$. Subsequently, $300 \mathrm{U} / \mathrm{ml}$ recombinant human IL-2 was added to the media. At day 14, CIK cells were harvested and analyzed for phenotype and cytotoxicity. All cells, reagents and materials were free of bacteria, mycoplasma or fungal contamination. The measured endotoxin levels were $<5$ endotoxin units.

Statistical analysis. Overall survival was estimated using the Kaplan-Meier method, and calculated from the date of diagnosis to the date of death from the disease or the last follow-up. Survival estimates were calculated by the Kaplan-Meier method using SPSS 19 software (IBM SPSS, Armonk, NY, USA). $\mathrm{P}<0.05$ was considered to indicate a statistically significant difference.

\section{Results}

Cases. All cases were confirmed to have advanced-stage disease based on clinical imaging and pathological studies, five $(62.5 \%)$ of which had lymph node metastasis, one $(12.5 \%)$ had pancreatic and adrenal gland invasion, one (12.5\%) had pancreatic and spleen invasion, and the remaining one $(12.5 \%)$ had invasion of the inferior vena cava according to the CT scan. The clinicopathological features of the eight cases are presented in Table I.

Surgery. Four cases received radical nephrectomy, one case was subjected to radical nephrectomy and splenectomy, 


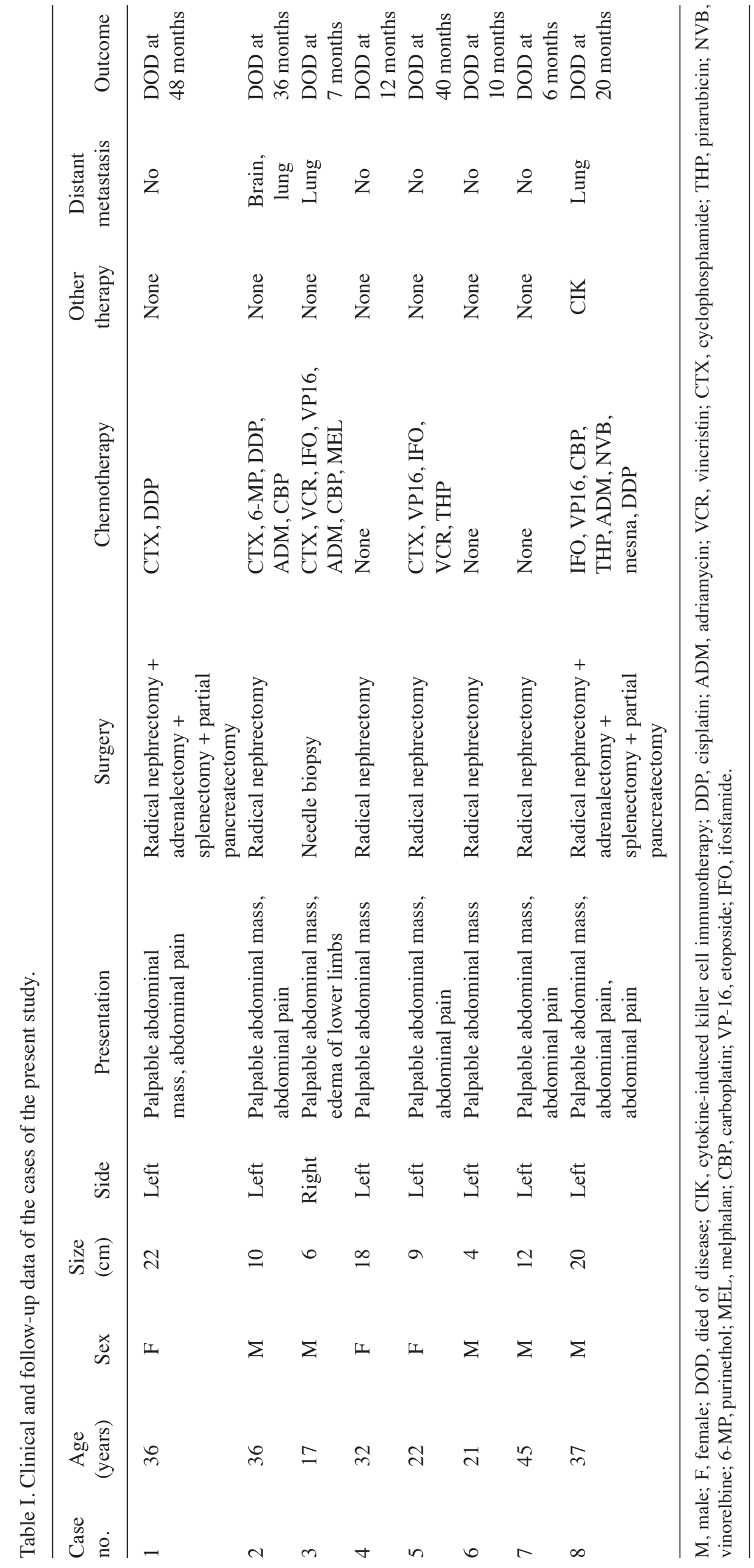


and two cases received radical nephrectomy combined with splenectomy and partial pancreatectomy. Lymph node dissection was also performed during the surgery. The tumor was inoperable in the patient with invasion of the inferior vena cava according to the CT scan, and only a needle biopsy was performed to confirm the diagnosis. There were no major intra-operative and post-operative complications, or post-operative mortality.

Histopathology. Pathological review and immunohistochemistry (IHC) were performed to confirm the diagnosis. Histology revealed small round cells with a high nuclear-to-cytoplasmic ratio with vaguely defined cytoplasmic borders in the tumor. Homer-Wright rosette formation was identified in the tumors of three patients. IHC revealed positivity for CD99, vimentin and neuron-specific enolase (NSE) in six cases (cases 1, 3, 4, 5, 6 and 7; 75\%), Wilms' tumor protein (WT-1) in two cases (cases 6 and 7; 25\%) and desmin in one case (case $5 ; 12.5 \%$ ); furthermore, five cases $(62.5 \%)$ were negative for cytokeratin (CK) and one (12.5\%) was focal positive (Fig. 2). Fluorescent in situ hybridization (FISH) analysis using a locus-specific EWS/FLI-1 fusion gene dual color break apart rearrangement probe was performed in one case, revealing a translocation of chromosomes 11 and 22, t(11;22) (q24;q12).

Adjuvant treatment. Of all the cases, five received adjuvant chemotherapy (Table I). Chemotherapeutic agents included cyclophosphamide (CTX), pirarubicin (THP), cisplatinum (DDP), vinorelbine (NVB), purinethol (6-MP), melphalan (MEL), adriamycin (ADM), carboplatin (CBP), vincristine (VCR), etoposide (VP-16), ifosfamide (IFO) and mesna.

As a novel treatment, CIK cell immunotherapy was offered to all of the patients, while only one patient decided to receive this immunotherapy with informed consent. This patient received two courses of carboplatin $(500 \mathrm{mg}$, d1) and docetaxel $(80 \mathrm{mg}, \mathrm{d} 2)$, and two cycles of CIK cell immunotherapy in parallel with chemotherapy. This patient received a median of $95 \times 10^{8} \mathrm{CIK}$ cells per cycle. No severe side-effects of the CIK cell immunotherapy were observed. After CIK was administered, the patient complained of less gastrointestinal adverse events compared with the patients receiving chemotherapy only.

Follow-up. Follow-up information was available for all of the patients, which included physical examination, complete blood count, renal and liver function tests, chest X-ray and CT scan of the abdomen. Of the eight cases, five had localized recurrence only, one had localized recurrence and distant metastasis, and two had distant metastasis only. Distant metastatic sites included the lung (three cases) and brain (one case). None of the patients received any salvage therapy, with the exception of alterations in the chemotherapy regimens and dosage. Follow-up of these patients ranged from 6-48 months, with a median of 16 months. Overall median survival was 20 months with a 3-year survival rate of $25 \%$. Of the seven cases who had surgery, the overall survival in four patients who received adjuvant chemotherapy was 36 months, compared with 10 months in the three patients without adjuvant chemotherapy (Fig. 3). The patient receiving CIK cell immunotherapy survived for 20 months.

\section{Discussion}

Although peripheral PNET may occur in any soft tissue, it rarely occurs in the genitourinary system (10). Since rPNET was first described in 1975, only 120 confirmed cases have been reported. rPNET frequently affects young adults (mean age, 26 years) and occurs more commonly in males (males/females, 1.5:1) (11,12). In the present study, the patients with advanced-stage rPNET were relatively young (median age, 34 years; range, 17-45 years), and a slight male predominance was found (males/females, 1.6:1). Compared with PNET originating from other sites, rPNET is more aggressive, with a five-year disease-free survival rate of $45-55 \%$ at the well-confined stage and only $20-30 \%$ at the advanced stage. The poor prognosis of patients with rPNET is due to its non-specific clinical presentation, tendency to metastasize and advanced stage at the time of identification in the majority of cases $(13,14)$. The present study focused on rPNET at the advanced stage, and the cases presented with non-specific symptoms and clinical signs, such as palpable abdominal mass (100\%), abdominal pain (62.5\%) and signs of metastasis. The overall median survival was 20 months with a three-year survival rate of $25 \%$, which was markedly lower compared with the previously reported overall median survival of 40 months with a 3-year survival rate of $60 \%$ in patients who had localized disease (5). Therefore, distinguishing rPNET from other kidney malignancies, and detecting it early, is crucial for the management and prognosis of patients with rPNET.

Although other small round-cell tumors, including neuroblastomas (NBs), synovial sarcomas, small cell carcinomas, lymphomas, Wilms' tumors, and so forth, render the renal tumor differentiation difficult, rPNET does have specific histological features, including small uniform round cells with dark nuclei, ill-defined cytoplasmic borders, and poorly-formed rosette-like structures (15). In addition, IHC is important for accurately diagnosing rPNET. Parham et al (4) reported that it is difficult to characterize rPNET without IHC. According to IHC, $84-100 \%$ of rPNETs are positive for CD99, a macrophage inhibitory cytokine (MIC-2) gene product $(14,16)$. In addition, IHC markers, including vimentin, NSE and S-100, may aid in the diagnosis of rPNET and differentiation from other tumor types; however, they are not pathognomonic. Molecular diagnostic markers, such as friend leukemia integration 1 (FLI-1) and WT-1, were found to be relatively specific in terms of the diagnosis of rPNET, and a previous study reported that $60 \%$ of rPNETs were positive for FLI-1 expression by IHC (17), indicating that this marker may aid in the differentiation of rPNET from other types of renal tumor. Within the cohort of the present study, the tumors of $37.5 \%$ of cases had a specific histological structure called 'Homer-Wright rosette', and IHC analysis revealed that $65 \%$ of cases were positive for CD99, vimentin and NSE. In addition, positivity for WT-1 was found in two cases (25\%) and for desmin in one case (12.5\%); Furthermore, five cases $(62.5 \%)$ were negative for CK, while one case $(12.5 \%)$ was focal positive. WT-1, a common marker for Wilms' tumor, has been used to rule out the diagnosis of PNET, while rPNETs may stain positive for WT-1. A cytogenetic study performed to detect the EWS/FLI-1 fusion gene revealed a translocation of chromosomes 11 and 22, $\mathrm{t}(11: 22)$ (q24:q12), in $>90 \%$ of 

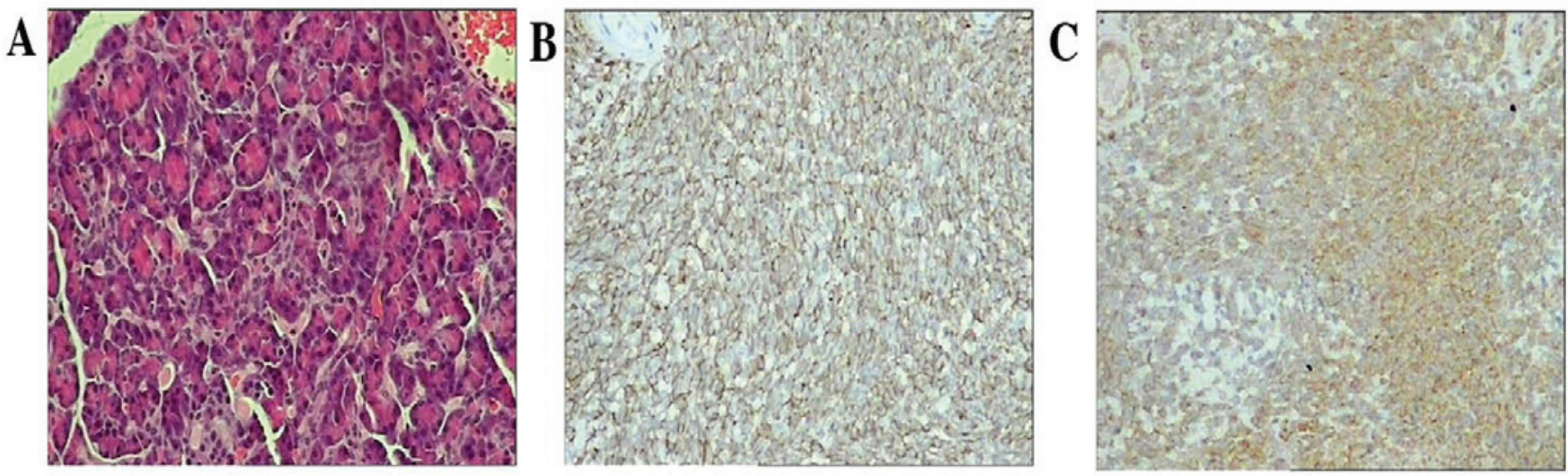

Figure 2. Histological and immunohistochemical images of a tumor sample from case no. 8. (A) Hematoxylin and eosin staining showed that the tumor cells formed rosette-like structures (magnification, x200). (B) The tumor cells were strongly positive for CD56 (magnification, x100). (C) The tumor cells were positive for CD99 (magnification, x100).

A

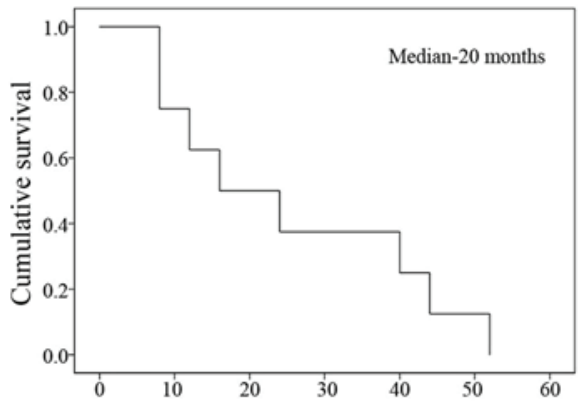

B

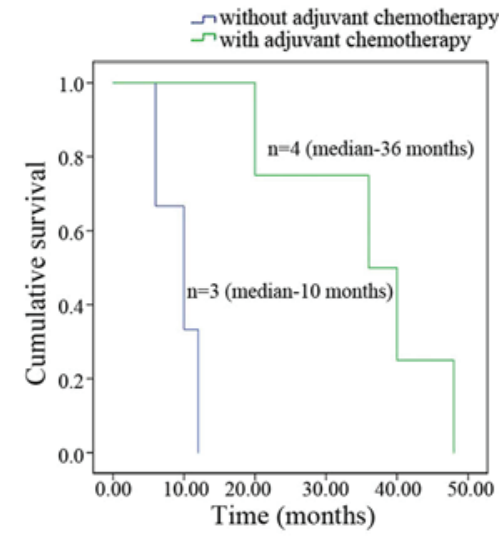

Figure 3. Survival estimates calculated by the Kaplan-Meier method. (A) Kaplan-Meier curve showing overall survival. (B) Kaplan-Meier curve showing overall survival curves in patients with adjuvant chemotherapy after surgery $(n=4)$ and without adjuvant chemotherapy $(n=3)$.

rPNET cases (18), while the use of FISH for the detection of the fusion gene may increase the specificity of this marker and decrease false-positive test results. In the present study, FISH detection was performed for one case, also revealing $\mathrm{t}(11 ; 22)(\mathrm{q} 24 ; \mathrm{q} 12)$ translocation.

At present, no definitive guidelines for the treatment of rPNET are available. For rPNET at the localized stage, surgical excision is the first choice, providing a greater survival advantage compared with any other singular treatment (5). However, the prognosis of rPNET at an advanced stage remains poor in spite of aggressive treatment by combination therapy, including surgery, chemotherapy and radiotherapy $(1,6)$. In the present study, seven cases received radical nephrectomy, of which three cases had surgical resection of other organs involved. Four cases received adjuvant chemotherapy and had a significantly better overall survival of 36 months, compared with an overall survival of 10 months in the three patients without adjuvant chemotherapy. However, severe gastrointestinal adverse events are usually found during chemotherapy. In an attempt to improve the survival of high-risk patients as well as their quality of life, CIK cell immunotherapy was planned after surgery in combination with the administration of intensive and multiple large-dose post-operative adjuvant chemotherapies. In the one patient who consented to the CIK cell treatment, survival for 20 months was achieved. Of note, the patient complained of less adverse events associated with chemotherapy. CIK cells are ex vivo activated lymphocytes with potent activity against various tumor types and minimal side-effects. Since CIK cells were first applied for renal cancer therapy in 1999 (19), the safety and efficacy of this immunotherapy has been confirmed and the most frequent adverse event is only mild, transient and easily controllable (20). The largest study of autologous CIK cell immunotherapy in metastatic RCC to date performed by Liu et al (9) found that CIK cell treatment significantly improved the prognosis of patients with metastatic RCC, while the prognosis was significantly improved for patients who received $\geq 7$ cycles of CIK infusions (9). CIK cell therapy is considered to have synergistic effects with conventional therapies, including chemotherapy or IL-2/IFN- $\alpha$ biotherapy in patients with RCC. CIK cells exert cytotoxic activities against solid tumors by specifically binding to target cells via cell surface adhesion molecule leukocyte function associated antigen-1 (LFA-1) to form cellular conjugates (21). Besides adhesion molecules, CIK cells express activating NK receptors, including NKG2D and DNAX accessory molecule-1, which leads to degranulation and activates T-cell receptor-independent tumor cell recognition and killing $(22,23)$. To the best of our knowledge, the present study was the first to report on the clinical application of CIK cell immunotherapy for the treatment of rPNET. The benefit of CIK cells and the possible synergy with targeted 
therapies for rPNET should be assessed in future studies using larger cohorts.

In conclusion, rPNET is a rare malignancy of the family of 'small round-cell tumors'. Patients with rPNET at the advanced stage have poor prognosis, and aggressive multimodality treatment, including surgical excision and chemotherapy, is recommended to manage these tumors. CIK cell immunotherapy may have the capacity to improve the prognosis and life quality of patients with rPNET. Further studies are required to validate the benefit of CIK cells and establish an appropriate immunotherapy protocol.

\section{Acknowledgements}

The authors would like to thank all of the patients enrolled in this study. This work was supported by the Medical Scientific Research Foundation of Guangdong Province (no. A117), the Fundamental Research Funds for the Central Universities (no. 16ykjc15) and the Natural Science Foundation of Guangdong Province (no. 2014A030310158).

\section{References}

1. de Alava E and Gerald WL: Molecular biology of the Ewing's sarcoma/primitive neuroectodermal tumor family. J Clin Oncol 18: 204-213, 2000

2. Mani S, Dutta D and De BK: Primitive neuroectodermal tumor of the liver: A case report. Jpn J Clin Oncol 40: 258-262, 2010.

3. Seemayer TA, Thelmo WL, Bolande RP and Wiglesworth FW: Peripheral neuroectodermal tumors. Perspect Pediatr Pathol 2: 151-152, 1975

4. Parham DM, Roloson GJ, Feely M, Green DM, Bridge JA and Beckwith JB: Primary malignant neuroepithelial tumors of the kidney: A clinicopathologic analysis of 146 adult and pediatric cases from the National Wilms' Tumor Study Group Pathology Center. Am J Surg Pathol 25: 133-146, 2001.

5. Thyavihally YB, Tongaonkar HB, Gupta S, Kurkure PA, Amare P, Muckaden MA and Desai SB: Primitive neuroectodermal tumor of the kidney: A single institute series of 16 patients. Urology 71: 292-296, 2008.

6. Chu WC, Reznikov B, Lee EY, Grant RM, Cheng FW and Babyn P: Primitive neuroectodermal tumour (PNET) of the kidney: A rare renal tumour in adolescents with seemingly characteristic radiological features. Pediatr Radiol 38: 1089-1094, 2008.

7. Kim JS, Chung IS, Lim SH, Park Y, Park MJ, Kim JY, Kim YG, Hong JT, Kim Y and Han SB: Preclinical and clinical studies on cytokine-induced killer cells for the treatment of renal cell carcinoma. Arch Pharm Res 37: 559-566, 2014.

8. Li H, Yu JP, Cao S, Wei F, Zhang P, An XM, Huang ZT and Ren XB: CD4+CD25+ regulatory $T$ cells decreased the antitumor activity of cytokine-induced killer (CIK) cells of lung cancer patients. J Clin Immunol 27: 317-326, 2007.

9. Liu L, Zhang W, Qi X, Li H, Yu J, Wei S, Hao X and Ren X: Randomized study of autologous cytokine-induced killer cell immunotherapy in metastatic renal carcinoma. Clin Cancer Res 18: 1751-1759, 2012.
10. Bartholow T and Parwani A: Renal primitive neuroectodermal tumors. Arch Pathol Lab Med 136: 686-690, 2012.

11. Ellinger J, Bastian PJ, Hauser S, Biermann K and Müller SC: Primitive neuroectodermal tumor: Rare, highly aggressive differential diagnosis in urologic malignancies. Urology 68: 257-262, 2006.

12. Lam JS, Hensle TW, Delbelenko L, Granowetter L and Tennenbaum SY: Organ confined primitive neuroectodermal tumour arising from the kidney. J Pediatr Surg 38: 619-621, 2003.

13. Rodríguez-Galindo C, Liu T, Krasin MJ, Wu J, Billups CA, Daw NC, Spunt SL, Rao BN, Santana VM and Navid F: Analysis of prognostic factors in ewing sarcoma family of tumors: Review of St. Jude Children's Research Hospital Studies. Cancer 110: 375-384, 2007.

14. Ellison DA, Parham DM, Bridge J and Beckwith JB: Immunohistochemistry of primary malignant neuroepithelial tumors of the kidney: A potential source of confusion? A study of 30 cases from the national Wilm's tumor study pathology center. Hum Pathol 38: 205-211, 2007.

15. Wada Y, Yamaguchi T, Kuwahara T, Sugiyama Y, Kikukawa H and Ueda S: Primitive neuroectodermal tumour of the kidney with spontaneous regression of pulmonary metastases after nephrectomy. BJU Int 91: 121-122, 2003.

16. Ravindra $\mathrm{S}$ and Kini U: Cytomorphology and morphometry of small round-cell tumors in the region of the kidney. Diagn Cytopathol 32: 211-216, 2005.

17. Risi E, Iacovelli R, Altavilla A, Alesini D, Palazzo A, Mosillo C, Trenta $\mathrm{P}$ and Cortesi E: Clinical and pathological features of primary neuroectodermal tumor/Ewing sarcoma of the kidney. Urology 82: 382-386, 2013.

18. Kumar R, Gautam U, Srinivasan R, Lal A, Sharma U, Nijhawan R and Kumar S: Primary Ewing's sarcoma/primitive neuroectodermal tumor of the kidney: Report of a case diagnosed by fine needle aspiration cytology and confirmed by immunocytochemistry and RT-PCR along with review of literature. Diagn Cytopathol 40 (Suppl 2): E156-E161, 2012.

19. Schmidt-Wolf IG, Finke S, Trojaneck B, Denkena A, Lefterova P, Schwella N, Heuft HG, Prange G, Korte M, Takeya M, et al: Phase I clinical study applying autologous immunological effector cells transfected with the interleukin-2 gene in patients with metastatic renal cancer, colorectal cancer and lymphoma. Br J Cancer 81: 1009-1016, 1999.

20. Jäkel CE, Hauser S, Rogenhofer S, Müller SC, Brossart P and Schmidt-Wolf IG: Clinical studies applying cytokine-induced killer cells for the treatment of renal cell carcinoma. Clin Dev Immunol 2012: 473245, 2012.

21. Pievani A, Borleri G, Pende D, Moretta L, Rambaldi A, Golay J and Introna M: Dual-functional capability of CD3+CD56+ CIK cells, a T-cell subset that acquires NK function and retains TCR-mediated specific cytotoxicity. Blood 118: 3301-3310, 2011.

22. Girardi M, Oppenheim DE, Steele CR, Lewis JM, Glusac E, Filler R, Hobby P, Sutton B, Tigelaar RE and Hayday AC: Regulation of cutaneous malignancy by gammadelta $\mathrm{T}$ cells. Science 294: 605-609, 2001.

23. Karimi M, Cao TM, Baker JA, Verneris MR, Soares L and Negrin RS: Silencing human NKG2D, DAP10 and DAP12 reduces cytotoxicity of activated $\mathrm{CD} 8+\mathrm{T}$ cells and NK cells. J Immunol 175: 7819-7828, 2005. 